\title{
ESTUDIO DEL ARTE PARIETAL PALEOLÍTICO DESDE LA PERSPECTIVA ARQUEOLÓGICA: VIEJOS FANTASMAS/ NUEVOS ENFOQUES
}

\author{
Study of the paleolithic parietal art from the archaeological perspective: \\ old ghosts / new approaches
}

\author{
Clara HERNANDO ÁLVAREZ* \\ Universidad de Salamanca \\ E-mail: clara85@usal.es
}

Fecha de recepción: 19-01-2010

Fecha de aceptación: 29-01-2010

RESUMEN: Esta comunicación pretende analizar los grafismos parietales paleolíticos desde la perspectiva arqueológica, dejando a un lado los vínculos estéticos de la historiografía tradicional. Se contrastarán las técnicas de análisis tradicional del arte paleolítico europeo con nuevas aplicaciones interdisciplinares, buscando un conocimiento arqueológico de las manifestaciones gráficas de las sociedades prehistóricas. Por último se propondrá un ensayo metodológico aplicando la Matriz Harris en la lectura y ordenación de los grafismos paleolíticos del panel central de la cueva de Llonín (Peñamellera Alta, Asturias)

Palabras Clave: Paleolítico, arte parietal, Prehistoria, Arqueología, metodología, estratigrafía, Matriz Harris, Llonín.

ABSTRACT: This paper aims to analyse the parietal palaeolithic graphic designs from an archaeological perspective, leaving aside the aesthetic ties of traditional historiography. Traditional European analysis techniques of palaeolithic rock art will be contrasted with new interdisciplinary applications, looking for an archaeological knowledge about the artistic graphic expression of prehistoric societies. Finally, a methodological trial will be suggested applying the Harris Matrix in the reading and arrangement (ordination) of the palaeolithic graphic designs of the central panel of Llonin's cave (Peñamellera Alta, Asturias). 
Keywords: Palaeolithic, Parietal art, Prehistory, Archaeology, Methodology, Stratigraphy, Harris Matrix, Llonín.

\section{INTRODUCCIÓN}

Durante mucho tiempo, el estudio de los grafismos paleolíticos ha sido una disciplina más cercana al análisis estético y artístico que a la investigación arqueológica y prehistórica. Las interpretaciones 'románticas' y casi fantásticas han relegado esta disciplina a un cajón de sastre, no demasiado elaborado, en el que todo tenía cabida (las teorías que aludían a una magia propiciatoria de la caza; las teorías que postulaban la reducción de los grafismos prehistóricos a elementos sexuales, o el chamanismo como justificación de los signos no identificables...) $)^{1}$. Actualmente, el avance de la multidisciplinariedad y la motivación de los jóvenes investigadores han permitido que se revisen planteamientos y concepciones decimonónicas de la metodología a aplicar en su estudio. Aún así, parece que aún queda mucho para hacer del arte paleolítico un elemento más del registro arqueológico de la Prehistoria. Tenemos los medios a nuestra disposición para que dichos acercamientos interpretativos lo sean desde un punto de vista científicamente argumentado. Es por ello que proponemos una lectura puramente arqueológica (en metodología y concepción) de los grafismos paleolíticos.

\section{LOS VIEJOS FANTASMAS DEL “ARTE” PALEOLÍTICO}

El concepto 'arte' que entiende y aplica nuestro mundo contemporáneo tiene una alta carga estética, creadora de una reacción emocional individual en el espectador, como la nacida en María Sanz de Sautuola, al contemplar en 1879 el techo de los bisontes de Altamira. Por ello, muchos investigadores se han posicionado frente a dicha conceptualización cargada de vínculos estéticos, prefiriendo hablar de pinturas y grabados rupestres o muebles, símbolos o imágenes ${ }^{2}$, 'representaciones materiales' o 'imágenes de tiempos glaciares'3. Para algunos de ellos', el uso del término 'arte' suponela 'proyección anacrónica' de una idea contemporánea aluniverso pasadodela Prehistoria. En las primeras décadas del siglo XX esta disciplina se adentra en el ámbito de la ciencia no sin dificultades, tras lograr superponerse al ideal de hombre prehistórico como un ser salvaje, recién salido de la animalidad 5 .

La metodología aplicada en los estudios del arte prehistórico surge de sus primeros descubridores, analizando la variabilidad formal de los grafismos en términos esencialmente cronológicos, cuyas convenciones estilísticas respondían a una época concreta, inmersa en una evolución progresiva y lineal del arte a lo largo del tiempo. Esta percepción evolutiva partirá de la 
labor de E. Piette, H. Breuil, H. Cartailhac, H. Alcalde del Río, etc., quienes elaboraron las primeras secuencias temporales a partir de la observación y búsqueda de paralelos formales en las grafías.

E. Piette $(1907)^{6}$ estableció un método de datación, denominado 'genético simple’, que consistía en el desarrollo lineal de la producción gráfica a lo largo del tiempo. Le seguiría H. Breuil en su afán de estructurar los grafismos prehistóricos en bloques cronológicos evolutivos, recorriendo los 'santuarios' desde los motivos más antiguos (más torpes) a los más recientes (del elaborado Magdaleniense, cuyo paradigma reside en Altamira). H. Breuil consideraba que la investigación sobre el significado era algo secundario y que lo más importante era el conocimiento de las obras de arte en sí mismas? Es por ello, que se centrará en el estudio cronológico de las manifestaciones, bajo la idea de una larga secuencia (40.000-10.000 a.C.) como pondría de reflejo en una obra posterior Quatre cents siècles d'art pariètal (1952), partiendo de la comparación con el arte mobiliar, el análisis de las superposiciones y de una concepción evolucionista del arte. Para H. Breuil, la evolución del grafismo prehistórico partía de la adquisición de dos parámetros o capacidades por parte del hombre; la conquista de la tercera dimensión y el enriquecimiento de las técnicas, desde los primeros grabados a la pintura polícroma?. Sin embargo, la secuencia formal que permitió a $\mathrm{H}$. Breuil definir diversos ciclos cronoestilísticos, no fue estructurada ni sistematizada, a excepción de los análisis referidos a la perspectiva ${ }^{10}$.

En cuanto al protocolo de actuación metodológica de los prehistoriadores de principios del siglo XX, éste se reducía a la elaboración de calcos directos o esbozos de las representaciones prehistóricas. H. Breuil muestra el paradigma de actuación, calcando las imágenes sobre el soporte pétreo o bien, cuando las condiciones de fragilidad no lo permitían, evitaba el contacto con el muro, realizando copias a mano alzada ${ }^{11}$. El cambio metodológico no se aprecia hasta los años 70, con el avance de la fotografía y su aplicación en la lectura del arte paleolítico, iniciadas por los prehistoriadores franceses (Aujoulat ${ }^{12}$, el GRAPP ${ }^{13}$, Lorblanchet ${ }^{14} \ldots$... Actualmente, las tecnologías a nuestro alcance han roto gran parte de los obstáculos de esta ciencia; y sin embargo, no se ha creado un protocolo sistemático y común a todos los investigadores.

Los trabajos durante más de un siglo han sido una combinación de descripciones formales, emociones subjetivas y analogías poco rigurosas. Los estudios del 'arte' paleolítico residían en los principios estéticos, definidos por la Historia del Arte, sin existir en los prehistoriadores una preocupación por la fórmula de metodologías y objetivos de la investigación apropiados para el estudio de los grafismos del Paleolítico como elemento representativo de los posibles intercambios y fenómenos de aculturación entre los grupos humanos, es decir, como cultura material $^{5}$. 
El cambio de paradigma ${ }^{16}$ vino de la mano de A. Laming-Emperaire $(1962)^{17}$ y A. Leroi-Gourhan $(1965)^{18}$, autores estructuralistas que valoraron las cavidades y sus conjuntos gráficos como unidades indisolubles y sincrónicas, en relación a un grupo social que debió establecer su hábitat en los vestíbulos de las cavidades decoradas. Estos autores establecieron un corpus muy completo, con una base estadística argumentada; sin embargo, actualmente la sistemática establecida es lo único que se mantiene de sus propuestas, siendo muy pocos los prehistoriadores que continúan aplicando sus interpretaciones estilísticas, como base de una cronología aproximada ${ }^{19}$.

La propia entidad de los grafismos paleolíticos, fijados en su lugar original, más que una ventaja, ha supuesto la descontextualización de muchos de ellos. No estando vinculados a los estratos horizontales de una excavación, la obtención de sus cronologías es complicada y una de las problemáticas más recurrentes en los análisis. Uno de los recursos primeramente señalados fue el estudio de las superposiciones de los grafismos ${ }^{20}$, sin embargo esta propuesta de investigación únicamente concedió un esquema rígido de cronologías relativas obtenidas a partir de ciertas figuras (algunas excepcionales), en el que se insertaron a posteriori, el resto de manifestaciones, como si de un puzzle se tratara ${ }^{21}$. Ahora bien, la herramienta estratigráfica, correctamente aplicada en el análisis de un panel rupestre, nos puede explicar el proceso de ejecución y el proceso gráfico, desarrollados en una cavidad. Esta metodología de lectura de estratigrafía parietal puede ser de gran ayuda cuando el investigador se encuentre frente a verdaderos palimpsestos como son, por ejemplo, el friso de los grabados del Gran Salón de Candamo (Asturias), el gran panel de Llonín (Asturias), o la cueva de la Griega de Pedraza (Segovia), donde las unidades gráficas y conjuntos gráficos se superponen sin ninguna reserva, a pesar de que el resultado sea un embrollo de trazos, casi imposibles de descifrar.

La superposición de diferentes fases gráficas, sincrónicas o diacrónicas sobre un panel, presenta una complicada deconstrucción si no existe una ayuda gráfica y estructurada. Proponemos por tanto, un ensayo metodológico aplicando la Matriz. Harris en la reconstrucción del panel principal de la cueva de Llonín (Peñamellera Alta, Asturias), lo que nos permitirá facilitar el análisis y la explicación de las relaciones de superposición entre las grafías; ayudándonos en la obtención de cronologías relativas ${ }^{22}$.

\section{ACERCAMIENTO A LA ESTRATIGRAFÍA ARQUEOLÓGICA}

"La estratigrafía es el estudio descriptivo de los estratos arqueológicos, su aparición, composición natural, sucesión y clasificación, con el objeto de ordenarlos en una secuencia cronológica"23. La estratigrafía es un concepto 
clave en la disciplina arqueológica, sin embargo, no fue hasta 1973, cuando Edward C. Harris formuló una simple estructura de representación y estudio de los niveles estratigráficos ${ }^{24}$. La construcción de dicha secuencia estratigráfica ha generado (hasta la actualidad) resultados óptimos en el análisis ar-

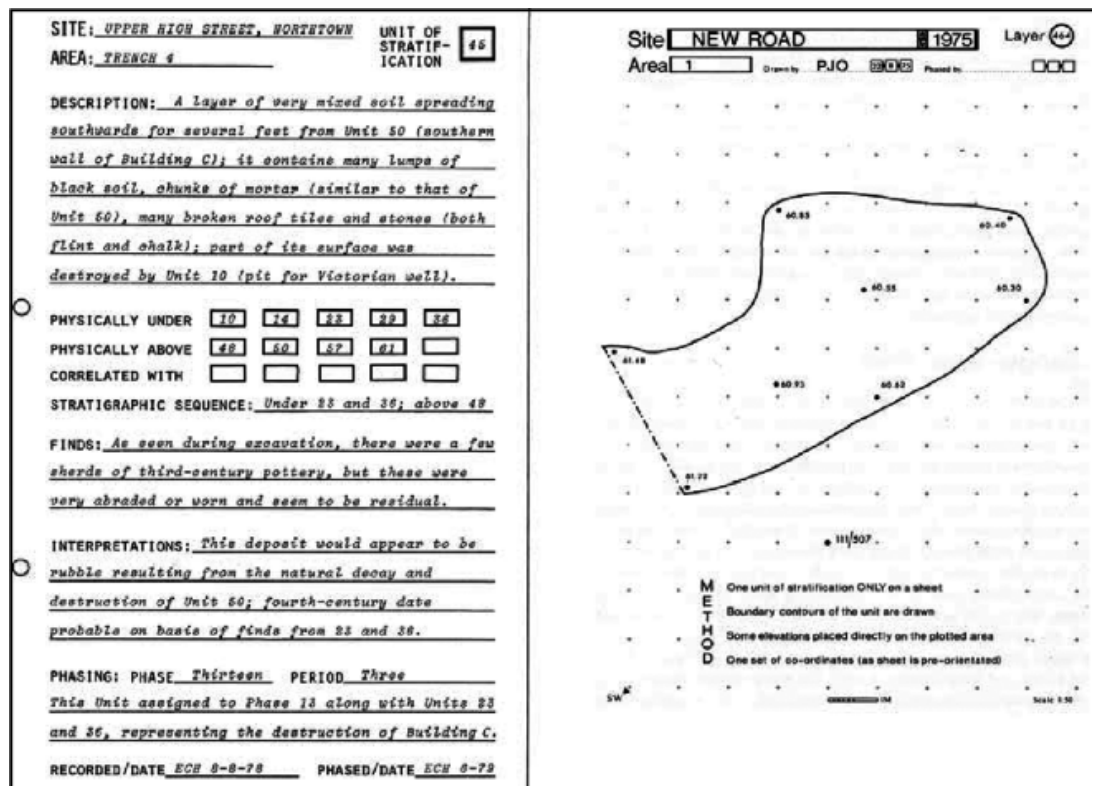

Lám. I. La ficha analítica del sistema Harris, a) delantera; b) trasera con el plano de estrato. (Harris 1979:96).

queológico, pues organiza la información mediante un diagrama, facilitando su lectura e interpretación. El diagrama de Harris funciona como un árbol genealógico, en el que se representan los diferentes tiempos: "antes", "después" y "durante" de la diacronía existentes entre unidades, acciones humanas o eventos naturales, representadas de forma abstracta mediante unidades estratigráficas a las que se asignan números diferenciadores ${ }^{25}$. Este sistema de registro arqueológico sustituyó a los métodos de excavación vigentes (como el método Wheeler), revolucionando las viejas representaciones bidimensionales (perfiles estratigráficos y planimetrías), que introdujeron como base fundamental la variable temporal o cuarta dimensión. A grandes rasgos, adaptaba los principios de la estratigrafía geológica a elementos antrópicos como son los niveles o estratos de una excavación arqueológica. La metodología estricta y sistemática es el telón de fondo de esta propuesta, que tiene como bases de ordenación las fichas de unidad estratigráfica (lám. I) y un elemento 
gráfico esquemático, la Matriz. Harris (lám. II).

El elemento esencial es la unidad estratigráfica (UE), «la plus petite unité de terrain situable antérieurement et postérieurement aux unités voisines par l'examen de ses limites physiques $\rangle^{26}$, que presenta un conjunto de variables propias, diferenciables del resto de unidades, que se unen en un esquema gráfico simplificado, denominado Matriz de Harris. En este diagrama se representan, mediante signos gráficos convencionales las relaciones básicas que pueden existir entre dos unidades estratigráficas.

-A es más reciente que (se superpone a) B.

-A es más antigua que (se infrapone a) $B$.

-A y B son unidades contemporáneas.

-A y B no tienen ninguna relación en común.

Cada unidad estratigráfica se define con un número y se enmarca en un recuadro; mientras que las relaciones que existen entre las mismas son señaladas mediante líneas conectoras. La unidad más reciente se ordena en la parte superior, sobre la unidad a la que se superpone. Las relaciones diacrónicas se significan mediante un trazo vertical que une dos UE; sin embargo, las unidades sincrónicas se disponen paralelas y se unen con un doble trazado horizontal. La única norma a cumplir es que los trazos conectores nunca deben de cruzarse.

Dichas relaciones fueron resumidas en una ley de sucesión estratigráfica por la cual "una unidad de estratificación arqueológica ocupa su lugar exacto en la secuencia estratigráfica de un yacimiento, entre la más baja (o más antigua) de las unidades que la cubren y la más alta (o más reciente) de todas las unidades a las que cubre, teniendo contacto físico con ambas y siendo redundante cualquier otra relación de superposición"”27. El objetivo de dicha formulación es la obtención de una datación relativa y secuencial de cada unidad analizada en relación a las demás, escapando a los datos cronológicos preexistentes o prejuicios estilísticos, que deberán ser contratados a posteriori en el diagrama resultante. Una vez identificada la secuencia estratigráfica y gracias a la ayuda de la información estilística o de observaciones cronotipológicas, se puede intentar formular una hipótesis de periodos de ejecución o construcción estructural ${ }^{28}$. Ésta debería ser la secuencia de actuación del investigador sistemático y científico. 


\section{NuEVOS ENFOQUES: LECTURA ESTRATIGRÁFICA DE LOS GRAFISMOS PALEOLÍTICOS}

Los grafismos paleolíticos se localizan en ocasiones en grandes conjuntos de superposiciones gráficas indescifrables, similares a la sucesión de estratos en un yacimiento arqueológico. Muchos autores abogan por una lectura estratigráfica parietal, quedando reducida a la búsqueda de secuencias donde las tipologías gráficas y estilismos intervienen de forma activa. La propuesta de E. Harris, que debería haber resuelto muchas dudas metodológicas en los investigadores del área, parece haber quedado relegada a la dimensión horizontal de las excavaciones. Sin embargo, actualmente, una nueva corriente denominada Arqueología de la Arquitectura ha 'enderezado' la matriz, para aplicarla a la lectura de los lienzos murarios de la Edad Media. Así, la edilicia medieval se ha dotado de un nuevo protocolo de actuación e interpretación de sus fuentes materiales, consiguiendo un bagaje instrumental y conceptual estrictamente arqueológico, bien diferenciado del empleado por otras disciplinas como la Historia del Arte o de la Arquitectura ${ }^{29}$. Conocidos son los trabajos de investigación y restauración de la catedral de Santa María de Vitoria-Gasteiz, en la que se ha experimentado la integración de la lectura estratigráfica de sus muros guiada por cronotipologías relativas ${ }^{30}$; los análisis en Santa Comba de Bande (Orense) $)^{31}$, San Juan Bautista de Baños (Palencia) ${ }^{32}$ o la iglesia de San Pedro de la Nave (Zamora).

¿Por qué no ayudarnos de este impulso para reivindicar la búsqueda científica de las cronologías relativas del grafismo paleolítico? ¿Por qué no aplicar una metodología sistemática y ordenada en el estudio de las unidades gráficas y del espacio decorativo prehistórico? De forma indirecta, muchos investigadores enuncian la importancia de la lectura estratigráfica, pero evitan la sistematización científica de su desarrollo en el trabajo de campo. Las herramientas con las que contamos hacen necesario el establecimiento de un protocolo de actuación común a los proyectos de investigación referidos al registro gráfico prehistórico.

C. Chippindale y P. Taçon ${ }^{33}$ (1993) fueron los primeros en proponer la aplicación del método de lectura Harris a los grafismos paleolíticos de Arnhem Land, en Australia, verificando arqueológicamente la secuencia temporal de los estilos gráficos elaborados para dicho sitio arqueológico. Tan sólo se ha constatado un intento metodológico posterior en la misma línea, realizado por J. H. Loubser $(1997)^{34} \mathrm{y}$ aplicado a los grabados rupestres de Tandjesberg (África del Sur) y del Abrigo de El ratón (Baja California, Mexico), clarificando a través del esquema gráfico la sucesión de escenas representadas en el registro arqueológico. Los trabajos de dichos investigadores han resaltado la necesidad de «clarifier les relations stratigraphiques d'un site et ainsi remettre en cause nos avis sur la structure, la conservation et l'agge relatif $\|^{35}$. 
Tomando su ejemplo y partiendo de la construcción cronológica del arte paleolítico como esencia de nuestro conocimiento, aplicaremos esta metodología en el análisis y comprensión del panel principal de la cueva de Llonín (Asturias), apuntando de antemano que se trata únicamente de una propuesta metodológica ex situ, realizada desde un conocimiento bibliográfico y del proceder técnico de las investigaciones.

\section{ENSAYO METODOLÓGiCO EN LLONÍN (AsTURIAS)}

La cueva de Llonín se eleva frente al pico de Peñamellera, a un kilómetro del pueblo de Llonín, en Asturias. Descubierta en 1957 por dos hermanos, Francisco y Manuel Monje, fue remodelada y utilizada como depósito y lugar de fermentación de quesos. Su arte prehistórico sería ocultado hasta 1970, cuando dichos artesanos abandonan la cavidad para establecer su industria en Panes, dando noticia de las decoraciones ${ }^{36}$.

El dispositivo gráfico de la cavidad se localiza en dos ámbitos diferentes. El primero de ellos es la Gran Sala, donde se ubican el denominado Panel de Entrada, Panel de la Sala, Panel del Cono Posterior y Panel Principal, en los que se grabaron y pintaron figuraciones de cérvidos, renos, bisontes y un antropomorfo; además de conjuntos gráficos compuestos de digitalizaciones, trazados rectilíneos y 'meandriformes' en ocre o negro. La segunda estancia decorada es La Galería, un estrecho conducto elevado en el que únicamente se han constatado conjuntos gráficos, sin atribución zoomorfa.

De todos ellos, el Panel Central de Llonín presenta numerosas superposiciones gráficas, que configuran una secuenciación del proceso de ejecución, desarrollada en una superficie que alcanza los 13 metros y que es apropiada para desarrollar nuestra propuesta metodológica. Los últimos datos han propuesto una secuencia de ejecución formada por 5 fases gráfi$\mathrm{Cas}^{37}$. No interpretaremos dicha contribución pues necesitaría un estudio en profundidad de la estratigrafía parietal; pero las numerosas superposiciones existentes, la hacen favorable al método presentado como un ejemplo de aplicación metodológica, no de interpretación arqueológica.

El protocolo de actuación propuesto deber ser realizado por un equipo científico (mínimo 3 personas), cuyas valoraciones y lecturas sean contrastadas. El trabajo a realizar se compone de diversas fases de análisis:

1. Comprensión espacial de la cavidad decorada. El investigador/es debe/n recorrer la caverna localizando sobre un plano, lo más detallado posible, las unidades topoiconográficas (UT) (áreas que presentan decoración) y las unidades compositivas (UC) (relación entre grafías que ocupan un mismo espacio figurativo definido como hornacina, panel...). El tránsito por la cavidad 
debe ser reflexivo y atento, dejando un hueco a lo desconocido e interrogándonos acerca de las representaciones ya documentadas.

2. Comprensión de los paneles de forma individual. A continuación, debe existir una primera lectura aproximativa a los grafismos a través de un pequeño croquis o boceto a mano alzada.

3. Registro gráfico y documentación. La documentación gráfica (fotográfica y realización de calcos) es una labor lenta y sistemática. No incidiremos en este apartado acerca de las metodologías de fotografía de material arqueológico, ni del tratamiento de las reproducciones informáticas y calcos digitales, si bien remitimos a los trabajos de C. Fritz y G. Tosello ${ }^{38}$, que desde el centro de investigación del CREAP en Toulouse, aportan los métodos de lectura y registro más avanzados. En cuanto a la documentación de las manifestaciones paleolíticas, ha de ser elaborada una ficha de análisis gráfico-estratigráfico en la que de forma ordenada y bajo un sistema de siglas, quede recogida toda unidad mínima de análisis que conforma la secuencia estratigráfica parietal. Dos son los conceptos utilizados: UNIDAD GRÁFICA (UG), elemento identificable individualmente ${ }^{39}$, de origen antrópico y paleolítico que puede ser definido como zoomorfo ${ }^{40}$; y CONJUNTO GRÁFICO (CG), que alude a diferentes formas que pudieron o no conformar una unidad, no pudiendo ser señalada ésta por la escasa relación entre los trazos, o por la mala conservación del dispositivo. UG y CG se numeran de forma consecutiva de izquierda a derecha en el panel, que anteriormente ha sido dividido en sectores (A, B, C... ), facilitando su organización.

Así, cada unidad mínima se analiza de forma meticulosa a través de una ficha de análisis gráfico-estratigráfico, anotando todas sus variables de análisis individual (técnica de ejecución, temática, formato, orientación, animación, grado de detalle, elementos anatómicos representados...) y de conjunto (relaciones asociativas entre las unidades gráficas o conjuntos gráficos). Las relaciones espaciales entre las grafías se anotarán mediante los términos de yuxtaposición amplia o estrecha y superposición, que responde a la existencia de asociaciones repetidas y aleatorias, cuyo análisis se desarrolla en la fase sucesiva ${ }^{41}$.

4. Documentación de las relaciones estratigráficas. En cada una de las fichas, la relación espacial establece una estratigrafía a pequeña escala, que junto al resto de unidades analizadas, convergen en un esquema o diagrama estratigráfico completo del panel. Los espacios en que se multiplican las superposiciones y cruces de líneas se denominan 'nudos de información'42 y necesitan de una observación lenta y concienzuda, para la cual podemos ayudarnos del tratamiento informático de las fotografías y calcos elaborados, si bien es siempre necesario volver a la cavidad para cerciorarnos de lo visto durante el análisis de gabinete. 


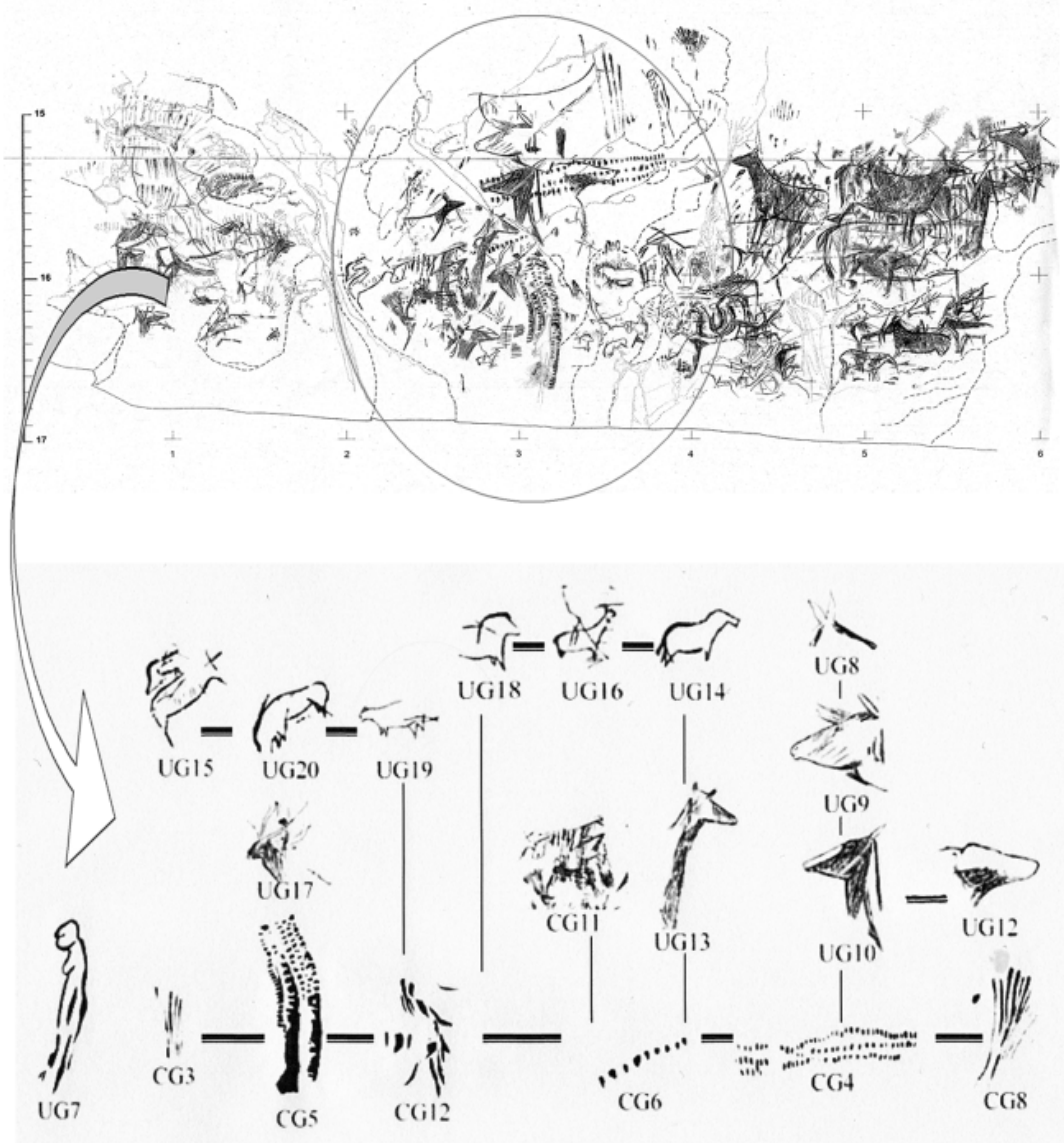

Lam II (arriba). Calco del panel principal de la cueva de Llonín (FORTEA et al., 2004:15-17, modificado).

Lám. 3. Lectura estratigráfica del sector B del panel central de Llonín, resumida a través del esquema Harris

La representación en imagen de la estratigrafía parietal es muy simple. La relación entre las unidades se establece mediante línea continua (que implica una relación de diacronía) y una doble línea (relación de sincronía y elementos análogos desde un punto de vista secuencial y estilístico). Como ejemplo, expresaremos de forma gráfica uno de los nudos de información 
(sector B) observados en el panel principal de Llonín. La lectura de sus superposiciones, ha sido representada mediante simples bocetos y líneas conectoras, las cuales aluden a la relación espacial existente entre las figuras rojas (digitaciones y puntuaciones), las representaciones de cérvidos en trazo estriado y los pequeños grabados zoomorfos localizados en la zona inferior decorada.

La Matriz. Harris nos permite, casi de un vistazo, comprender la secuencia temporal. La línea de conjuntos gráficos inferior (CG2, 3, 5, 12, 6...) coincide con los grafismos de pintura roja (puntuaciones, digitalizaciones...), mientras que la UG7 se ha identificado como un antropomorfo femenino ejecutado en pintura roja lineal, que aparece como elemento aislado del conjunto.

Algunas de las representaciones en ocre son cortadas y superpuestas por grabados zoomorfos, como es el caso de UG17, un prótomo de cáprido de gran cornamenta y técnica de grabado estriado sobre el CG5; las representaciones de cuadrúpedos indeterminados UG19 y UG20, grabado en trazo simple y que se superpone al CG12. Estos ejemplos aluden a diferentes etapas de desarrollo gráfico, sin determinar cuánto tiempo separa una de otra. Por tanto, la Matriz. Harris favorece la lectura e interpretación del proceso de decoración gráfica prehistórica.

Otra información aportada será la referente a la secuencia de ejecución, en la que se inscriben fases de decoración gráfica sincrónicas, que determinan una sucesión entre las mismas. De ellas, destacan las unidades gráficas 10, 9 y 8. La UG10 es una cabeza de cérvido mediante grabado estriado, que se superpone al CG4 de pinturas rojas. Sobre UG10 se realizó un prótomo de cérvido orientado a la izquierda, e infrapuesto a UG8, una cabeza de cérvido ejecutada en trazado estriado. Este ejemplo materializa la secuencia de ejecución del autor o los autores del lienzo decorado, indicando su intencionalidad y disposición ante la obra gráfica.

5. Creación de un diagrama estratigráfico completo para cada panel. El ejemplo propuesto recoge tan sólo 20 registros gráficos, sin embargo, la lectura del panel completo abarcaría más de 60 unidades de análisis. La construcción de la secuencia completa nos informará de los períodos de ejecución gráfica (en términos de cronología relativa) y de los posible procesos de desarrollo gráfico.

6. Interpretación y construcción de referentes cronotipológicos. Sólo terminada la secuencia estratigráfica, pueden incluirse en el análisis global del dispositivo, los elementos estilísticos y analogías formales, favoreciendo la creación de tiempos estilísticos absolutos, que pueden ponerse en relación con la ocupación de la cavidad en época paleolítica o con relaciones de aculturación y contacto entre grupos humanos de cronología afín. 


\section{CONCLUSIONES}

La estratigrafía, base científica de la Arqueología, debe ser también la base primera de la lectura y comprensión de los grafismos prehistóricos; previa al estudio analógico y estilístico de los dispositivos. La unión de ambos, nos permitirá obtener lecturas cronotipológicas desde una metodología científica y sistemática, imprescindible para la realización de hipótesis interpretativas sobre sus autores o su funcionalidad. El concepto está presente en los investigadores, pero no se ha establecido un protocolo de actuación común en las intervenciones arqueológicas, imprescindible si se quiere adquirir un conocimiento global de la materia y más aún de la sociedad prehistórica que es su autora. Un protocolo de actuación madurado por los organismos científicos debería recoger la sistemática de la Matriz. Harris, como vehículo de ordenación e interpretación de los datos, siendo además, un modo de registro gráfico perdurable en el tiempo y ajeno a las lecturas, interpretaciones y modas historiográficas de sus estudiosos. Al fin y al cabo, nuestra labor como investigadores tiene fecha de caducidad, pero la conservación de nuestro pasado debe ser siempre un valor conjugado en tiempo presente.

\section{REFERENCIAS BIBLIOGRÁFICAS}

ALCALDE DEL RÍO, H.; BREUIL, H. y SIERRA, L. (1911), Les cavernes de la region cantabrique. Mónaco.

ALCOLEA GONZÁLEZ, J. J. y BALBÍN BERHMANN, R. (2005), “Testigos del frío. La fauna en el arte rupestre paleolítico del interior peninsular" en Geomorfología y Patrimonio en la Península Ibérica y el entorno mediterráneo. ADEMA. Soria. (p. 547-566).

ALCOLEA GONZÁLEZ, J. J. y BALBÍN BERHMANN, R. (2006), Arte paleolítico al aire libre. El yacimiento rupestre de Siega Verde, Salamanca. Arqueología en Castilla y León. Memorias, 16.

AUjOUlAT, N. (1987), Le Relevé des Oeuvres Pariétales Paléolithiques. Enregistrement et Traitement des Données, Documents d'Archéologie Française, 9, Éditions de la Maison des Sciences de l'Homme. París.

AZKARATE GARAI-OLAUN A. (2001), "Análisis de la evolución histórico constructiva de la catedral de Santa María de Vitoria-Gasteiz (Aplicación de la 'Arqueología de la Arquitectura' a un modelo complejo)" en Actas del V Congreso de Arqueología Medieval Española, Valladolid, 1999, Volumen 1. (p.177-190).

BAHN, P. (1988), Images of the Ice Age. Facts on File. Londres.

BELTRÁN, A. (2002), Mito, misterio y sacralidad. La pintura prehistórica aragonesa. Biblioteca Aragonesa de Cultura. Zaragoza. 
BERENGUER, M. (1979), El arte parietal prehistórico de la Cueva de Llonín (Peñamellera Alta).-Asturias. Instituto de Estudios Asturianos (del Consejo Superior de Investigaciones Científicas). Caja de Ahorros de Asturias.

BREUIL, H. (1952), Quatre cents siècles d'art parietal. Montignac. Centre d'Etudes de la Documentation Préhistorique.

CABALlERO ZOREDA L., (2001), "Buscando unha Partida de Nacemento. Arqueoloxía da Arquitectura en Santa Comba de Bande (Orense)" en Lethes 3. (p. 58-75).

CABALLERO ZOREDA L. y FEIJOO S. (1998), "La iglesia altomedieval de San Juan Bautista en Baños de Cerrato (Palencia)" en Archivo Español de Arqueología, 71. (p. 181 242).

CABALLERO ZOREDA L.; ARCE SÁINZ F. y UTRERO AGUDO Mª A. (1999), Iglesia altomedieval de Santa Comba de San Torcuato, Bande (Orense).Lectura de paramentos, CSIC, Madrid (manuscrito).

CARANDINI, A. (1984), Arqueología y cultura material. Mitre. Barcelona. Barcelona.

CARANDINI, A. (1997), Historias en la tierra. Manual de excavación arqueológica. Crítica.

CHAPA BRUNET, T. (2000), "Nuevas tendencias en el estudio del Arte Prehistórico" en Arqueoweb, 2, 3. Departamento de Prehistoria, Universidad Complutense de Madrid. En línea: http://www.ucm.es/info/arqueoweb/numero2_3/articulo2_3_CHAPA.htm. (23-04-2007)

CHIPPINDALE, C. y TAÇON, P. (1993), «Two old painted Panels from Kakadu: Variation and sequence in Arnhem Land Rock Art" en STEINBRING, J.; WATCHMAN, A.; FAULSTICH, P. y TAÇON, P.S.C. (Eds.), Time and Space: Dating and Spacial Considerations Rock Art Research. Occasional AURA Publication, 8. Melbourne, (p. 32-56).

CLOTTES, J. y LEWIS-WILLIAMS, D. (2001), Los chamanes en la Prehistoria. Ariel Prehistoria. Barcelona.

CONKEY, M. W. (1997), "Beyond Art and between the caves: thinking about context in the interpretive process" en CONKEY, M. W. (Ed.), Beyond Art. Pleistocene Image and Symbol. San Francisco, California. (p. 343-367).

DESACHY, B. (2005), "Du temps ordonné au temps quantifié : application d'outils mathématiques au modèle d'analyse stratigraphique d'Edward Harris » en Bulletin de la Société Préhistorique Français, 102(4). (p. 729-740).

FERNÁNDEZ MARTÍNEZ, V. M. (2000), Teoría y Método de la Arqueología. Editorial Síntesis (2a ed.) (p. 129).

FORTEA, J. ; RASILLA, M. y RODRÍGUEZ OTERO, V. (2004), “L'art pariétal et la séquence archéologique paléolithique de la grotte de Llonín (Peñamellera Alta, Asturias, Espagne)" en Prébistoire, Art et Sociétés, 59. (p. 7-29). 
FRITZ, C. et TOSELLO, G. (2007), "The hidden meaning of forms: methods of recording paleolithic parietal art" en Journal of archaeological method and theory, 14 (1). (p. 4880).

GÁRATE, D. (2006), Análisis y caracterización de los conjuntos parietales en grafías zoomorfas punteadas. Una expresión pictórica propia del Paleolitico Superior Cantábrico. Tesis Doctoral, Noviembre de 2006. Universidad de Cantabria. Santander.

GARCÍA DÍEZ, M. y EGUIZABAL, J. (2008), La cueva de Venta Laperra. El grafismo parietal paleolitico y la definición de territorios gráficos en la región cantábrica. Karrantza Harana. Diputación Foral de Bizkaia.

GROUPE DE REFLEXION SUR L'ART PARIETAL PALEOLITHIQUE (GRAPP). (1993), L'art Pariétal Paléolithique: Techniques et méthodes d'étude. Ministère de l'Enseignement Supérieur et de la Recherche. Comité des travaux historiques et scientifiques.

HARRIS, E. D. (1979), Principles or Archaeology Stratigraphy. Academia Press. Londres (2 ed. 1989).

HARRIS, E. D. (1991), Principios de estratigrafía arqueológica. Crítica. Barcelona. (1ª ed. 1989 Academic Press Limited, Londres).

KUHN, T. (1990), La estructura de las revoluciones cientificas. Fondo de Cultura Económica. Mexico.

LAMING-EMPERAIRE, A. (1962), La signification de l'art rupestre paléolithique. Méthode et applications. Université de Paris. Editions. A et J. Picard et Cie. París.

LEROI-GOURHAN, A. (1965), Prehistorie de l'Art Occidental. Mazenod. París.

LORBLANCHET, M. (1995). Les Grottes ornées de la Prébistoire, Nouveaux Regards, Éditions Errance, París.

LORBLANCHET, M. (1999), La naissance de l'art. Genèse de l'art prébistorique dans le monde. Éditions Errance. París.

LOUBSER, J. H. N. (1997), «Utilisation des diagrammes de Harris dans l'enregistrement, la conservation, et l'interprétation des peintures rupestres » en INOR A, 18. (p. 1421).

MILETO, C. y VEGAS, F. (2003), "El análisis estratigráfico constructivo como estudio previo al proyecto de restauración arquitectónica: metodología y aplicación” en $A r$ queología de La Arquitectura, 2. (p. 189-196).

PIETTE, E. (1894), « Notes pour servir à l'Historire de l'Art Primitif » en L'Anthropologie. Burdin et Cie. Angers.

PIETTTE, E. (1907), L’art pendant l'age du renne. Masson de Paris. París.

QUIRÓS CASTILLO, J. A. (2002), “Arqueología de la arquitectura en España” en Arqueología de la arquitectura, 1. (p. 27-38). 
REINACH, S. (1903) "L'art et la magie á propos des peintures et des gravures de l'age du renne" en L'Anthropologie, 14. (p. 257-266).

\section{NOTAS}

* Titular de una beca de investigación del Ministerio de Ciencia e Innovación, dentro del programa FPU (Formación del Profesorado Universitario).

${ }^{1}$ REINACH, S. (1903) 'L'art et la magie á propos des peintures et des gravures de l'age du renne” en L'Anthropologie, 14. (p. 257-266). LEROI-GOURHAN, A. (1965), Prehistorie de l'Art Occidental. Mazenod. París. CLOTTES, J. y LEWIS-WILLIAMS, D. (2001), Los chamanes en la Prehistoria. Ariel Prehistoria. Barcelona.

2 LORBLANCHET, M. (1999), La naissance de l'art. Genèse de l'art préhistorique dans le monde. Éditions Errance. París.

${ }^{3}$ BAHN, P. (1988), Images of the Ice Age. Facts on File. Londres.

${ }^{4}$ BAHN, P. (1988), op. cit.; CONKEY, M. W. (1997) "Beyond Art and between the caves: thinking about context in the interpretive process" en CONKEY, M. W. (Ed.), Beyond Art. Pleistocene Image and Symbol. San Francisco, California. (p. 343-367); LORBLANCHET, M. (1999), op. cit.; y BELTRÁN, A. (2002), Mito, misterio y sacralidad. La pintura prehistórica aragonesa. Biblioteca Aragonesa de Cultura. Zaragoza.

${ }^{5}$ PIETTE, E. (1894), «Notes pour servir à l’Historire de l'Art Primitif » en L'Anthropologie. Burdin et Cie. Angers. (p. 1).

${ }^{6}$ PIETTE, E. (1907), L’art pendant l'age du renne. Masson de Paris. París.

${ }^{7}$ ALCALDE DEL RÍO, H.; BREUIL, H. y SIERRA, L. (1911) Les cavernes de la region cantabrique. Mónaco.

${ }^{8}$ BREUIL, H. (1952), Quatre cents siècles d'art parietal. Montignac. Centre d'Etudes de la Documentation Préhistorique.

${ }^{9}$ BREUIL, H. (1952), op.cit. (p. 18).

${ }^{10}$ BREUIL, H. (1952), op. cit.

${ }^{11}$ ALCALDE DEL RÍO, H.; BREUIL, H. y SIERRA, L. (1911), op. cit.

${ }^{12}$ AUJOULAT, N. (1987), Le relevé des oewvres pariétales paléolithiques. Enregistrement et traitement des fonnées, Documents d'Archéologie Française, 9, Éditions de la Maison des Sciences de l'Homme. París.

${ }^{13}$ GROUPE DE REFLEXION SUR L'ART PARIETAL PALEOLITHIQUE (GRAPP). (1993), L'art Pariétal Paléolithique: Techniques et méthodes d'étude. Ministère de l'Enseignement Supérieur et de la Recherche. Comité des travaux historiques et scientifiques.

${ }^{14}$ LORBLANCHET, M. (1995). Les grottes ornées de la Prébistoire, Nouveaux Regards, Éditions 
Errance, París.

${ }^{15}$ CARANDINI, A. (1984), Arqueología y cultura material. Mitre. Barcelona.

${ }^{16}$ KUHN, T. (1990), La estructura de las revoluciones cientificas. Fondo de Cultura Económica. Mexico.

${ }^{17}$ LAMING-EMPERAIRE, A. (1962), La signification de l'art rupestre paléolithique. Méthode et applications. Université de Paris. Editions. A et J. Picard et Cie. París.

${ }^{18}$ LEROI-GOURHAN, A. (1965), Prebistorie de l'Art Occidental. Mazenod. París.

${ }^{19}$ ALCOLEA GONZÁLEZ, J. J. y BALBÍN BERHMANN, R. (2005), “Testigos del frío. La fauna en el arte rupestre paleolítico del interior peninsular" en Geomorfología y Patrimonio en la Peninsula Ibérica y el entorno mediterráneo. ADEMA. Soria. (p. 547-566); ALCOLEA GONZÁLEZ, J. J. y BALBÍN BERHMANN, R. (2006), Arte paleolitico al aire libre. El yacimiento rupestre de Siega Verde, Salamanca. Arqueología en Castilla y León. Memorias, 16.

${ }^{20}$ BREUIL, H. (1952), op. cit.

${ }^{21}$ CHAPA BRUNET, T. (2000), "Nuevas tendencias en el estudio del Arte Prehistórico" en Arqueoweb, 2, 3. Departamento de Prehistoria, Universidad Complutense de Madrid. En línea: http://www.ucm.es/info/arqueoweb/numero2_3/articulo2_3_CHAPA.htm (23-042007).

${ }^{22}$ LOUBSER, J. H. N. (1997), «Utilisation des diagrammes de Harris dans l'enregistrement, la conservation, et l'interprétation des peintures rupestres » en INOR A, 18. (p. 15).

${ }^{23}$ FERNÁNDEZ MARTÍNEZ, V. M. (2000), Teoría y Método de la Arqueología. Editorial Síntesis $\left(2^{\mathrm{a}}\right.$ ed.) (p. 129).

${ }^{24}$ HARRIS, E. D. (1979), Principles or Archaeology Stratigraphy. Academia Press. Londres(2a ed. 1989).

${ }^{25}$ CARANDINI, A. (1997), Historias en la tierra. Manual de excavación arqueológica. Crítica. Barcelona. (p. 83).

${ }^{26}$ DESACHY, B. (2005), "Du temps ordonné au temps quantifié : application d'outils mathématiques au modèle d'analyse stratigraphique d'Edward Harris » en Bulletin de la Société Prébistorique Français, 102(4). (p. 730).

${ }^{27}$ HARRIS, E. D. (1991), Principios de estratigrafía arqueológica. Crítica. Barcelona. (1 a ed. 1989, Academic Press Limited, Londres). (p. 58).

${ }^{28}$ Cfr. MILETO, C. y VEGAS, F. (2003), "El análisis estratigráfico constructivo como estudio previo al proyecto de restauración arquitectónica: metodología y aplicación" en $A r$ queología de La Arquitectura, 2. (p. 192).

${ }^{29}$ QUIRÓS CASTILLO, J. A. (2002), "Arqueología de la arquitectura en España” en $A r$ queología de la arquitectura, 1. (p. 31).

${ }^{30}$ AZKARATE GARAI-OLAUN A. (2001), “Análisis de la evolución histórico constructiva 
de la catedral de Santa María de Vitoria-Gasteiz (Aplicación de la 'Arqueología de la Arquitectura' a un modelo complejo)" en Actas del V Congreso de Arqueología Medieval Española, Valladolid, 1999, Volumen 1. (p.177-190).

${ }^{31}$ CABALLERO ZOREDA L.; ARCE SÁINZ F. y UTRERO AGUDO Mª . A. (1999), Iglesia altomedieval de Santa Comba de San Torcuato, Bande (Orense).Lectura de paramentos, CSIC, Madrid (manuscrito). CABALLERO ZOREDA L., (2001), "Buscando unha Partida de Nacemento. Arqueoloxía da Arquitectura en Santa Comba de Bande (Orense)" en Lethes 3. (p. 58-75).

32 CABALLERO ZOREDA L. y FEIJOO S. (1998), "La iglesia altomedieval de San Juan Bautista en Baños de Cerrato (Palencia)" en Archivo Español de Arqueología, 71. (p.181-242).

${ }^{33}$ CHIPPINDALE, C. y TAÇON, P. (1993), «Two old painted Panels from Kakadu: Variation and sequence in Arnhem Land Rock Art" en STEINBRING, J.; WATCHMAN, A.; FAULSTICH, P. y TAÇON, P.S.C. (Eds.), Time and Space: Dating and Spacial Considerations Rock. Art Research. Occasional AURA Publication, 8. Melbourne, (p. 32-56)

${ }^{34}$ LOUBSER, (1997), op. cit.

${ }^{35}$ LOUBSER (1997), ídem.

${ }^{36}$ BERENGUER, M. (1979), El arte parietal prehistórico de la Cueva de Llonín (Peñamellera Alta).Asturias. Instituto de Estudios Asturianos (del Consejo Superior de Investigaciones Científicas). Caja de Ahorros de Asturias.

${ }^{37}$ FORTEA, J. ; RASILLA, M. y RODRÍGUEZ OTERO, V. (2004), “L'art pariétal et la séquence archéologique paléolithique de la grotte de Llonín (Peñamellera Alta, Asturias, Espagne)" en Prébistoire, Art et Sociétés, 59. (p. 7-29).

${ }^{38}$ FRITZ, C. et TOSELLO, G. (2007), “The hidden meaning of forms: methods of recording paleolithic parietal art" en Journal of archaeological method and theory ,14 (1). (p. 48- 80).

${ }^{39}$ GARCÍA DÍEZ, M. y EGUIZABAL, J. (2008), La cueva de Venta Laperra. El grafismo parietal paleolítico y la definición de territorios gráficos en la región cantábrica. Karrantza Harana. Diputación Foral de Bizkaia. (p. 33).

${ }^{40}$ GÁRATE, D. (2006), Análisis y caracterización de los conjuntos parietales en grafias zoomorfas punteadas. Una expresión pictórica propia del Paleolitico Superior Cantábrico. Tesis Doctoral, Noviembre de 2006. Universidad de Cantabria. Santander. (p. 44).

${ }^{41}$ La superposición repetida tiene lugar entres dos o más unidades gráficas identificables bajo una misma especie zoomorfa (cérvido-cérvido), mientras que la superposición aleatoria alude a la coincidencia espacial entre dos conjuntos gráficos o dos o más unidades gráficas identificadas con especies animales diferentes (cáprido-bisonte).

${ }^{42}$ MILETO, C. y VEGAS, F. (2003), “El análisis estratigráfico constructivo como estudio previo al proyecto de restauración arquitectónica: metodología y aplicación” en Arqueología de La Arquitectura, 2. (p. 192). 